\title{
Influencia de los programas de educación financiera sobre el comportamiento de los jóvenes: una revisión de literatura
}

Influence of financial education programs on the behavior of young people: a literature review

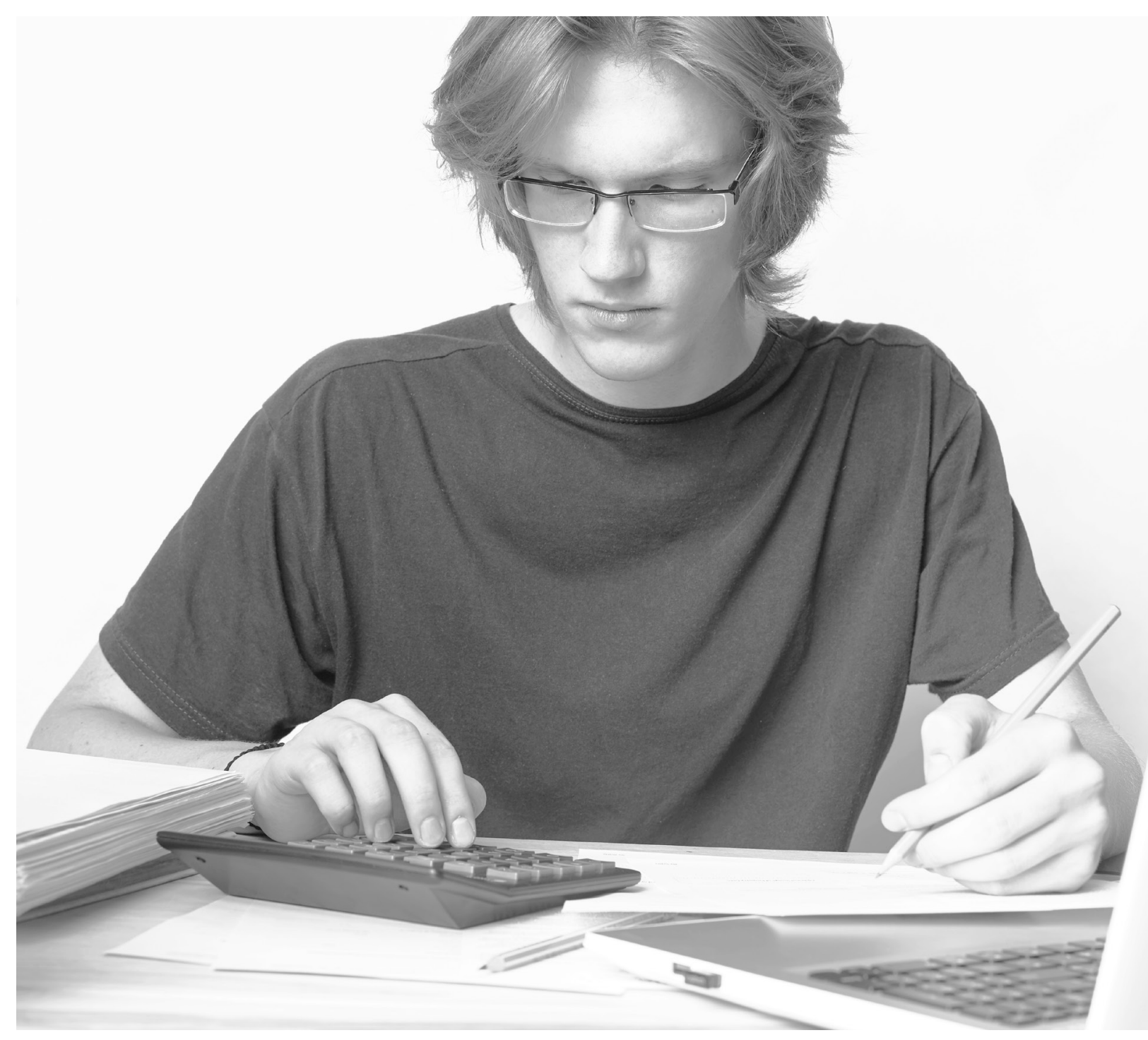




\title{
Influencia de los programas de educación financiera sobre el comportamiento de los jóvenes: una revisión de literatura ${ }^{1}$ Influence of financial education programs on the behavior of young people: a literature review
}

\author{
Karen Rocio Plata-Gómez², Jose Alonso Caballero-Márquez³
}

Artículo recibido en marzo 16 de 2020; artículo aceptado en abril 20 de 2020

\begin{abstract}
Este artículo puede compartirse bajo la licencia Licencia Creative Commons Atribución-NoComercial-Compartirlgual 4.0 Internacional y se referencia usando el siguiente formato: Plata-Gómez, K. R. y Caballero-Márquez, J. A. (2020). Influencia de los programas de educación financiera sobre el comportamiento de los jóvenes: una revisión de literatura. I+D Revista de Investigaciones, 15 (2), 18-27. DOl: https://doi.org/10.33304/revinv.v15n2-2020002
\end{abstract}

\begin{abstract}
Resumen
Dada la evidente necesidad de educar económica y financieramente a la población, se han implementado en diferentes partes del mundo estrategias y programas que permiten desarrollar los conocimientos y comportamientos financieros de los jóvenes. Sin embargo, existen pocos estudios que permitan extraer evaluaciones significativas del impacto de dichos programas sobre la transformación de los hábitos y comportamientos de los jóvenes en materia financiera. Por esta razón, este artículo busca reconocer la dinámica de publicación de dichos estudios, así como identificar, a través de los resultados de las diferentes investigaciones, los principales comportamientos financieros que logran desarrollar los jóvenes, a partir de los programas de educación financiera implementados en instituciones educativas en el ámbito global. Los resultados de esta investigación revelan que la implementación de estos programas genera cambios positivos sobre algunos de los comportamientos financieros de los jóvenes, principalmente sobre su comportamiento de ahorro y planificación para la jubilación, lo que les permite tomar decisiones financieras asertivas, y mejorar así su calidad de vida y la de sus familias.
\end{abstract}

Palabras clave: Alfabetización financiera, educación financiera, enseñanza secundaria, plan de estudios.

\begin{abstract}
Due to the evident need to educate the population economically and financially, strategies and programs have been implemented in different parts of the world, that allow the development of financial knowledge and the behavior of young people. However, few studies allow us to extract significant evaluations of the impact of these programs on the

${ }^{1}$ Artículo de revisión, con enfoque cualitativo, resultado de un proyecto de investigación en curso, perteneciente al área de ciencias sociales, subárea de ciencias de la educación, desarrollado en el grupo de investigación Finance \& Management, el grupo de investigación Estudios en Economía Aplicada y Regulación y el Grupo d e Estudio e Investigación en Tecnologías y Educación, en la Universidad Industrial de Santander, de la ciudad de Bucaramanga (Colombia). Dirección: carrera 27 calle 9. PBX: 6344000. Fecha de inicio: noviembre de 2019.

${ }^{2}$ Ingeniera industrial. Grupo de Investigación Finance \& Management, Universidad Industrial de Santander, Bucaramanga, Colombia. Dirección: carrera 27 calle 9. PBX: 6344000. ORCID ID: https://orcid.org/0000-0001-8871-6377. Correo electrónico: karengomez920@gmail.com.

${ }^{3}$ Magíster en Ingeniería Industrial. Grupo de Investigación Finance \& Management, Universidad Industrial de Santander, Bucaramanga, Colombia. Dirección: carrera 27 calle 9. PBX: 6344000. ORCID ID: https://orcid.org/0000-0002-8482-6381. Correo electrónico: josecaballeromrqz@gmail.com.
\end{abstract}


transformation of the habits and behaviors of young people in financial matters. Therefore, this article seeks to recognize the dynamics of publication of these studies, as well as to identify, through the results of the different investigations, the main financial behaviors that young people manage to change through financial education programs implemented in educational institutions globally. The results of this research reveal that the implementation of these programs generates positive changes in some of the financial behaviors of young people, mainly on their saving behavior and planning for retirement. The development of this behavior allows young people to make assertive financial decisions, thus improving their quality of life and that of their families.

Keywords: Curriculum, financial education, financial literacy, secondary education.

\section{Introducción}

Debido al rápido crecimiento en tecnología e innovación, la oferta de productos y servicios financieros ha aumentado en número, complejidad y alcance (Jayaraman et al., 2018; Liu et al., 2019). Esto conduce a nuevas oportunidades de crecimiento económico e inclusión financiera, pero también se asocia con numerosos riesgos, no solo entre los adultos, sino también entre los jóvenes, ya que estos últimos son particularmente vulnerables, debido a su deficiente nivel de educación económica y financiera, tanto regional como mundialmente (Amagir et al., 2018; Fabris y Luburic, 2016; Lusardi y Mitchell, 2011). Esto lo demuestran los resultados de las pruebas PISA 2012 en cuanto a la competencia de alfabetización financiera, la cual refleja el escaso conocimiento que poseen los jóvenes latinoamericanos respecto a los conceptos básicos financieros. En términos de planificación financiera, menos del $30 \%$ de los estudiantes puede calcular una tasa de interés simple (OECD, 2014).

Para el caso específico de Colombia, los resultados de la prueba son bastante desalentadores. Entre los países evaluados, Colombia obtuvo la puntuación media más baja frente a esta competencia, con una diferencia de 225 puntos respecto a Shanghái, China, que tuvo la puntuación media más alta de todas $(603,3)(\mathrm{OECD}$, 2014). Según estos resultados, más de la mitad de los alumnos colombianos se encuentran por debajo del nivel básico en esta competencia; situación que genera gran preocupación y demuestra la imperiosa necesidad de educar económica y financieramente a la población, lo que recientemente ha sido foco de atención entre los investigadores, ya que es uno de los principales factores que contribuyen al crecimiento económico y al desarrollo (Liu et al., 2019; Supanantaroek et al., 2017).

Lo anterior se puede soportar desde la literatura, ya que múltiples autores coinciden en que la desinformación, el comportamiento financiero irresponsable y las capacidades y habilidades financieras deficientes son las principales causas de la aparición de deudas, sobrecostos y desigualdad social (Amagir et al., 2018; Fabris y Luburic, 2016; Supanantaroek et al., 2017). Tal situación conduce a problemas de estabilidad y bienestar financiero, tanto a nivel individual como a nivel global. Frente a esto, la OCDE/ INFE (2019) reconoce que se deben mejorar los niveles de alfabetización financiera, y hace un llamado a los gobiernos, específicamente de América Latina y el Caribe, a promover el diseño e implementación de estrategias nacionales para educar económica y financieramente a la población.

En este sentido, a través de los años, en el ámbito mundial se han desarrollado e implementado en diferentes instituciones educativas múltiples programas y estrategias de educación financiera para los jóvenes. Sin embargo, hay pocos estudios que permitan extraer afirmaciones concluyentes o evaluaciones significativas del impacto de dichos programas sobre la transformación de los hábitos y comportamientos de los individuos en materia financiera (Acosta-Medina et al., 2020; Asobancaria, 2016; Cordero y Pedraja, 2019; Lusardi y Mitchell, 2014). Por tal motivo, esta revisión de literatura busca reconocer la dinámica de publicación de dichos estudios, y a partir de estos identificar los principales comportamientos financieros que logran cambiar o desarrollar los jóvenes a través de los programas de educación financiera implementados en instituciones educativas en el ámbito global. Lo anterior, con el objetivo de consolidar dicha información y aportar a la literatura existente sobre esta temática.

\section{Metodología}

\section{Tipo de estudio}

Esta investigación tiene un enfoque cualitativo, con un diseño de revisión documental, dado que busca estudiar e identificar los principales comportamientos financieros que desarrollan los jóvenes a través de los programas de educación financiera implementados en instituciones educativas alrededor del mundo, a partir del análisis de los resultados de las diferentes investigaciones encontradas en la literatura. El estudio es de tipo no experimental y longitudinal, porque valora aspectos de la documentación científica y analiza la trazabilidad de la literatura desde los orígenes de las publicaciones referentes a esta temática de investigación. 


\section{Procedimiento}

Para la presente revisión de literatura se realiza una búsqueda enfocada en los principales comportamientos financieros que logran cambiar los jóvenes en educación secundaria con edades entre los 15 y los 18 años, a través de los programas de educación financiera implementados en instituciones educativas alrededor del mundo. Lo anterior, mediante el uso de los portales de búsqueda Scopus e ISI Web of Science (WoS).

Adicionalmente, para complementar la información, se revisan aquellas referencias bibliográficas más citadas en la literatura consultada, con el fin de incorporarlas en la revisión. Posteriormente, el total de documentos encontrados se analiza mediante el uso del software Vantage Point. Por último, mediante lectura crítica, se seleccionan los artículos considerados de mayor relevancia y afinidad a la temática de investigación, y, a partir de los resultados de estos estudios, se realiza un análisis de literatura.

\section{Fundamentación teórica}

\section{Educación financiera}

Se refiere al proceso de información, asesoramiento o instrucciones a través del cual las personas mejoran su comprensión y conocimiento sobre los conceptos y riesgos financieros (Aprea et al., 2016; Fabris y Luburic, 2016). Lo anterior con el objetivo de desarrollar en las personas la confianza, la motivación y las habilidades que les permitan aplicar esos conocimientos y conceptos adquiridos en una gran variedad de contextos financieros; por ejemplo, para inducir a tomar decisiones responsables e informadas sobre planificación financiera, acumulación de riqueza, deudas, pensiones e inversión, con lo que se mejora el bienestar financiero de los individuos y la sociedad, y se propicia una mayor participación en la vida económica (Cordero y Pedraja, 2019; Förster et al., 2019; Liu et al., 2019).

\section{Alfabetización financiera}

La alfabetización consiste en la habilidad de leer y escribir una lengua especifica (Arango, 2018). A partir de este concepto, múltiples autores definen la alfabetización financiera como la habilidad o capacidad mínima de leer y escribir lo que se expresa en términos financieros, así como de entender su aplicación procurando un bienestar económico y financiero personal (Amagir et al., 2018; Cameron et al., 2014; Liu et al., 2019). Estas habilidades requieren ser desarrolladas y estimuladas desde etapas tempranas, a través de la educación financiera, ya que de este modo es posible influir significativamente en su mejoramiento, con lo que se logra que las personas adquieran la capacidad de cuestionarse y desarrollar un punto de vista crítico respecto a qué, cómo y cuándo gastar, invertir y ahorrar su dinero (Atkinson y Messy, 2012; Cordero y Pedraja, 2019; Ergün, 2018).

\section{Comportamiento financiero}

Se refiere a la manera en que reaccionan o actúan las personas, a partir de algún conocimiento previo, frente a las diversas situaciones que involucran la administración del dinero. De acuerdo con esta definición, se pueden distinguir tres componentes clave que permiten promover cambios en los comportamientos financieros (Amagir et al., 2018):

Conocimientos: Para saber cómo comportarse, es necesario estar informado adecuadamente sobre el dominio que se refiere al comportamiento previsto. Sin embargo, el efecto del conocimiento sobre los cambios en el comportamiento es relativamente limitado. Por lo tanto, deben tenerse en cuenta otros dos aspectos.

Habilidades: Para poder cambiar el comportamiento de una persona, es necesario que esta aprenda en primer lugar a controlar las habilidades operativas que se refieren al dominio de estos comportamientos; por ejemplo, cómo lidiar con el presupuesto.

Actitudes: Para poder aplicar algo fuera del contexto en el que se ha aprendido, es necesario tener autoeficacia y desarrollar la motivación necesaria para hacerlo.

La Figura 1 permite ilustrar la relación existente entre los conceptos definidos, y explica cómo el comportamiento financiero está asociado con la alfabetización financiera, y estos, a su vez, están influenciados por los niveles de educación e ingresos de los individuos (Ahmad et al., 2014).

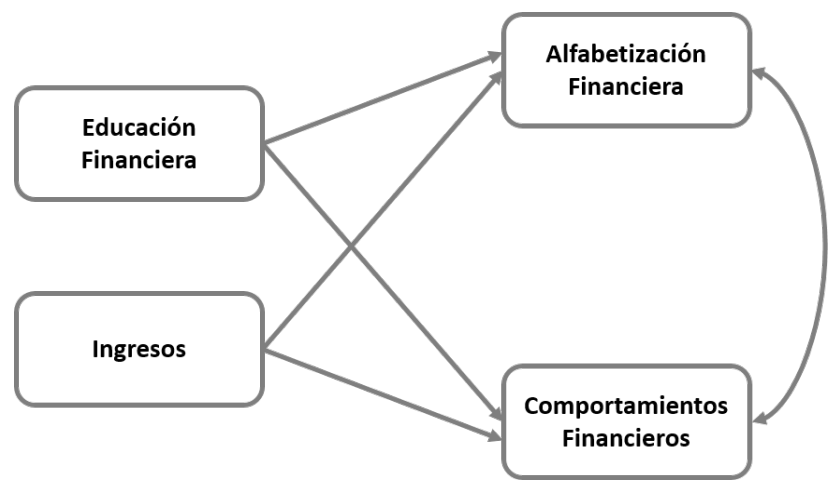

Figura 1. Esquema de la relación de conceptos del marco teórico. Fuente: (Ahmad et al., 2014).

\section{Programas de educación financiera}

El método más poderoso para mejorar la educación financiera de los jóvenes es aumentar la cobertura de las finanzas personales en el currículo escolar, o designar 
la educación económica y financiera como materia obligatoria, lo que debe promoverse desde el Gobierno hacia las instituciones educativas (Jang et al., 2014). Sin embargo, independientemente del enfoque que decidan emplear para implementar estos programas en dichas instituciones, el resultado que deben perseguir es el mismo, ya que deben enfocarse esencialmente en contribuir con la generación de cambios en los conocimientos, comportamientos y actitudes sobre temas financieros por parte de los estudiantes, así como propiciar cambios en su bienestar financiero (Asobancaria, 2016).

Del mismo modo, según la OECD (2005), estos programas deben ser diseñados para satisfacer las necesidades y el nivel de conocimientos financieros de la población $y$, además, a través de su implementación deben poder identificar cómo los estudiantes prefieren recibir esta información.

Adicionalmente, se recomienda realizar todo lo anterior en línea con las principales temáticas de los programas, que deben incluir conceptos financieros básicos (ahorros, presupuestos, impuestos, etc.), entendimiento de productos (servicios bancarios, seguros, pensiones, etc.) y riesgos asumidos (riesgos, seguridad y análisis financiero) (Asobancaria, 2016).

\section{Resultados}

En primer lugar, se presenta un análisis bibliométrico de la totalidad de artículos encontrados, y posteriormente, un análisis de revisión de literatura con los artículos seleccionados para estudio.

\section{Análisis bibliométrico}

Se plantea una ecuación de búsqueda con los términos que representan directamente los aspectos más relevantes del tema que se pretende abarcar. En tal sentido, se procede a incluir en la ecuación los términos "financial education", "financial literacy", "financial literacy education" y "financial-literacy education", con el fin de hallar artículos relacionados específicamente con el tema de educación o alfabetización financiera. En este caso, se incluyen entre comillas los términos, denotando que se requiere la presencia exacta de estos en los artículos, sin alteraciones de ningún tipo.

Adicionalmente, se agregan a la estructura de la ecuación los términos program*, "learning strategy", "teaching strategy", "teaching and learning strategies" y "curriculum", a fin de encontrar artículos relacionados con la implementación de un currículo educativo, plan de estudio, programa o metodología relacionado con el proceso de formación integral de los jóvenes en las instituciones educativas.
Así mismo, es necesario que la ecuación también contenga los términos behavio*, skill* y capabilit*, esperando encontrar artículos que, además, incluyan información referente a los comportamientos, habilidades y/o capacidades que estos jóvenes puedan transformar. Para esto, se emplea la función asterisco $\left(^{*}\right)$, con el objetivo de contar con las diferentes variaciones de estos términos en la búsqueda (como "behavior" o "behaviour", "skill" o "skills" y "capability" o "capabilities"). Por último, se agregan los términos "high school", "secondary education"y "secondary", con el fin de enfocar los resultados de la búsqueda en el área de la educación media o secundaria. En la Tabla 1 se presentan las ecuaciones de búsqueda propuestas tanto para la base de datos Web of Science como para Scopus.

Tabla 1

Ecuación de búsqueda

\begin{tabular}{cl}
\hline $\begin{array}{c}\text { Base de } \\
\text { datos }\end{array}$ & \multicolumn{1}{c}{ Ecuación } \\
\hline Web of & ALL= (("Financial education" OR "Financial literacy" \\
Science & OR Financial literacy education" OR "Financial- \\
& Literacy education") AND (programs OR "learning \\
& strategy" OR "teaching strategy" OR "teaching and \\
& learning strategies" OR curriculum) AND (behavio* \\
& OR skill* OR capabilit*) AND ( "high school" OR \\
& "secondary education" OR secondary)) \\
Scopus & TITLE-ABS-KEY (("Financial education" OR \\
& "Financial literacy" OR Financial literacy education" \\
& OR "Financial-Literacy education") AND (program* \\
& R "learning strategy" OR "teaching strategy" OR \\
& "teaching and learning strategies" OR curriculum) \\
& AND (behavio* OR skill* OR capabilit*) AND ("high \\
& school" OR "secondary education" OR secondary)) \\
\hline
\end{tabular}

Fuente: Autores.

El total de resultados obtenidos con esta búsqueda es de 104 documentos (33 de Web of Science y 71 de Scopus), entre los que se incluyen tipos de documentos como artículos, libros y revisiones. Esta búsqueda se realiza teniendo en cuenta todos los años de publicación disponibles en cada base de datos (2001-2020), sin restricciones de idioma o áreas de investigación.

A partir de estos documentos se realiza el análisis bibliométrico que se presenta en las siguientes líneas, el cual permite conocer la dinámica de publicación en esta temática en el ámbito mundial, considerando los campos de autores, países, palabras clave, principales áreas de investigación y años con mayor número de publicaciones.

En primer lugar, analizando el número de artículos publicados por año, sobre los estudios e investigaciones de la implementación de programas de educación financiera y su influencia en el comportamiento financiero de los jóvenes, se identifica una tendencia creciente en el número de publicaciones para los últimos 10 años, 
lo que confirma la vigencia actual de esta temática de investigación (ver Figura 2).

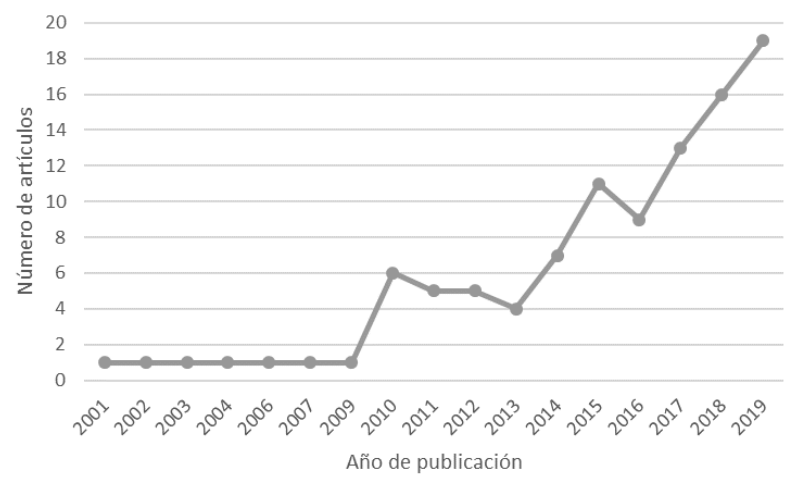

Figura 2. Publicaciones por año. Fuente: Autores utilizando el software Vantage Point.

Por su parte, la Figura 3 muestra los países con mayor número de publicaciones hasta la fecha, resaltando a Estados Unidos como el país con mayor número de producciones científicas sobre el tópico en cuestión. Lo anterior, dado que las primeras investigaciones sobre el tema fueron de origen estadounidense. Además de esto, múltiples autores confirman que casi todos los estudios que evalúan la efectividad de los programas de educación financiera se refieren a Estados Unidos (Cordero y Pedraja, 2019; Lusardi y Mitchell, 2011).

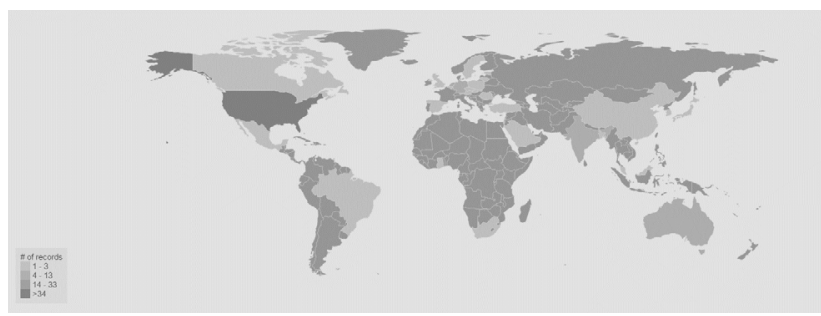

Figura 3. Países con mayor número de publicaciones. Fuente: Autores utilizando el software Vantage Point.

Así mismo, en la Figura 4 se presenta el número de publicaciones por año y país, y en ella se ratifica cómo desde el año 2001 hasta el 2009 está temática solo se trató en los Estados Unidos.

Adicionalmente, podemos confirmar a través de este análisis cómo efectivamente en el ámbito mundial se refleja un bajo nivel de estudios e investigaciones que abordan esta temática, resaltando principalmente a gran parte de Europa, África y América Latina.

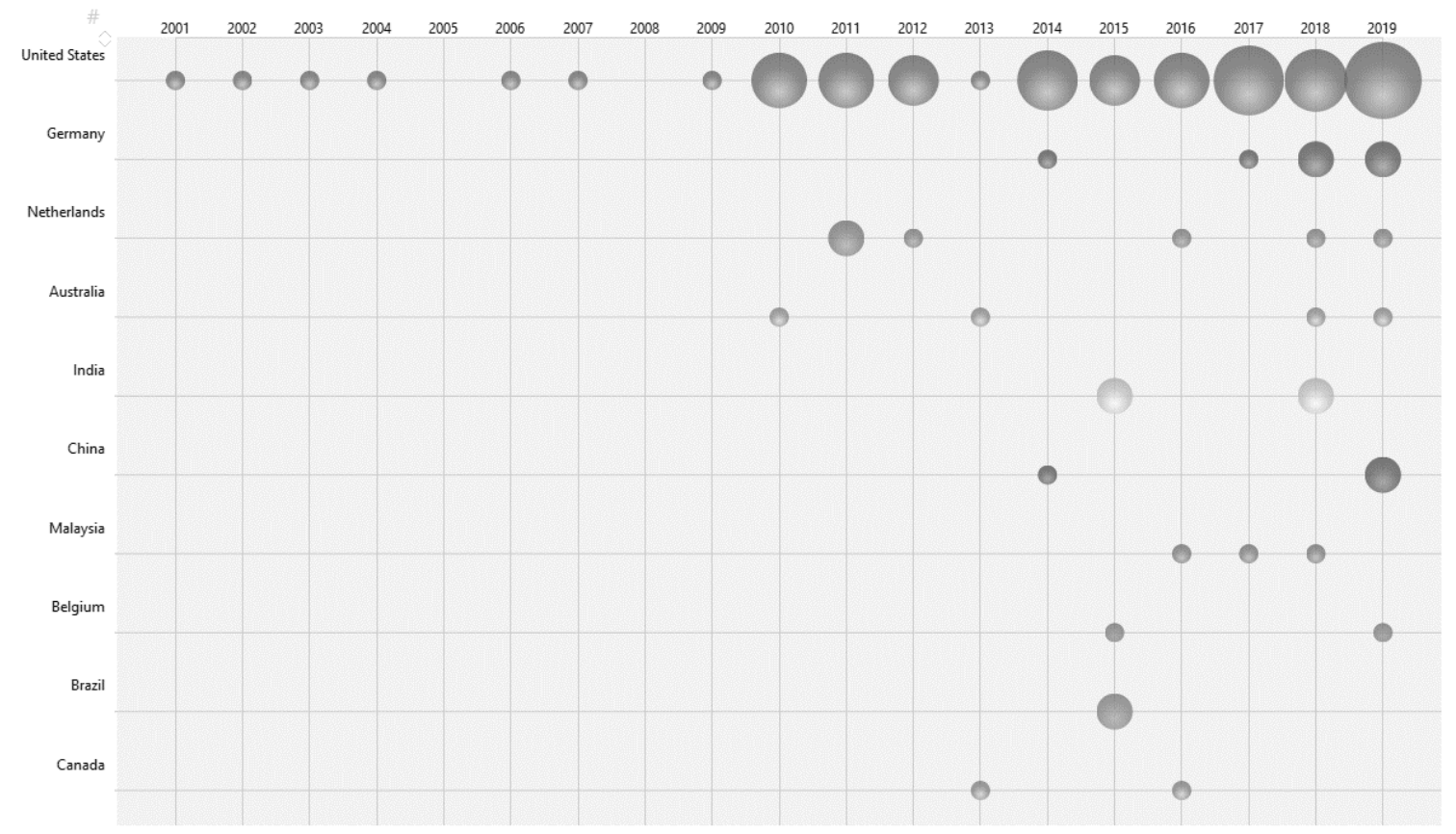

Figura 4. Países con mayor número de publicaciones por año. Fuente: Autores utilizando el software Vantage Point.

Por otro lado, con la ayuda del software Vantage Point, se genera la nube de palabras presentada en la Figura 5 , para lo cual solo se consideran los 10 términos clave más empleados por los autores en sus investigaciones. A partir de los resultados, se observa que los 5 términos más destacados son "financial literacy", "financial education", "financial behaviour", "economics education" y "finance".

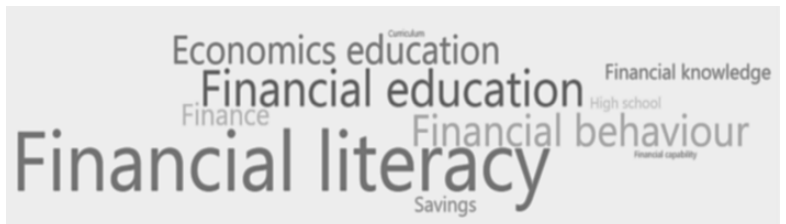

Figura 5. Nube de palabras clave. Fuente: Autores utilizando el software Vantage Point. 
En cuanto a la Figura 6, se logran identificar los autores más representativos y relevantes en esta temática de investigación. En tal sentido, según se observa, los 5 autores más citados son Lusardi, Mitchell, Huston, Alessie y Van Rooij.

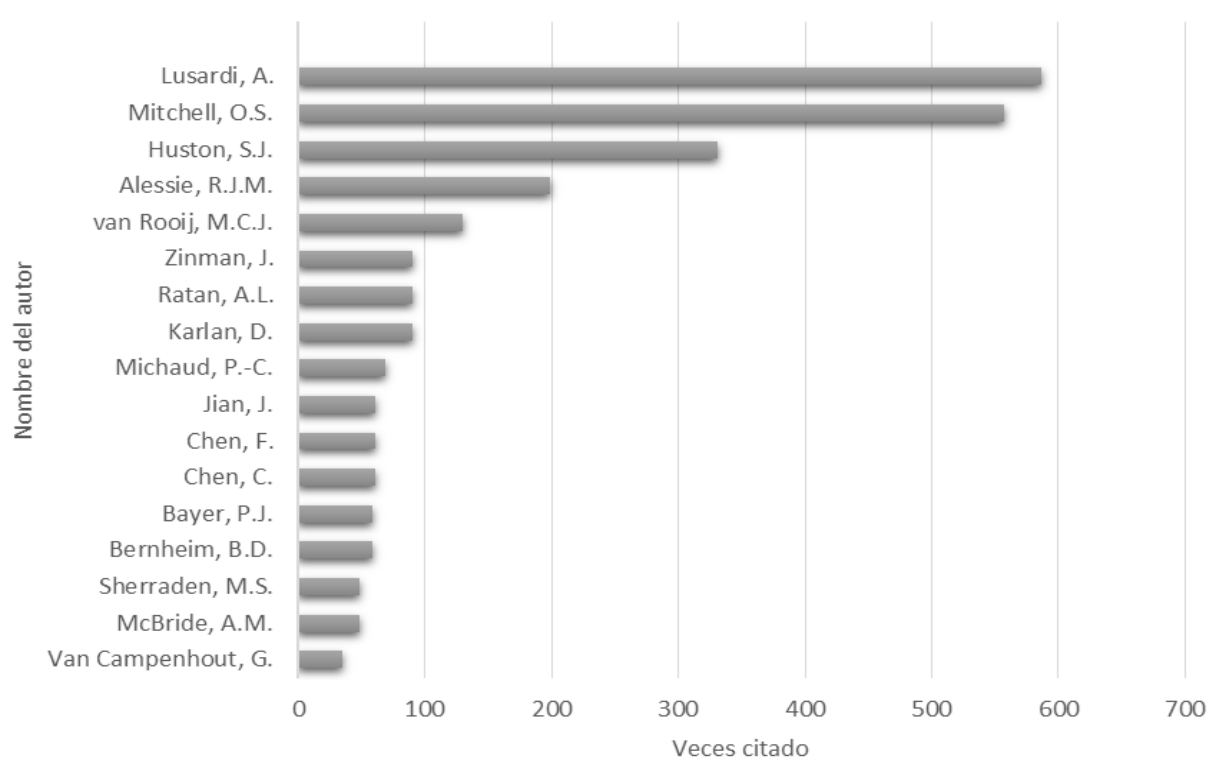

Figura 6. Número de citaciones por autor. Fuente: Autores utilizando el software Vantage Point.

Finalmente, se crea una aduna entre autores, como se ilustra en la Figura 7, teniendo en cuenta los más citados, con el objetivo de analizar la conexión entre ellos. De este modo, se observar como la mayoría de autores ha colaborado entre sí para realizar la producción de uno o más artículos científicos en esta temática de investigación.

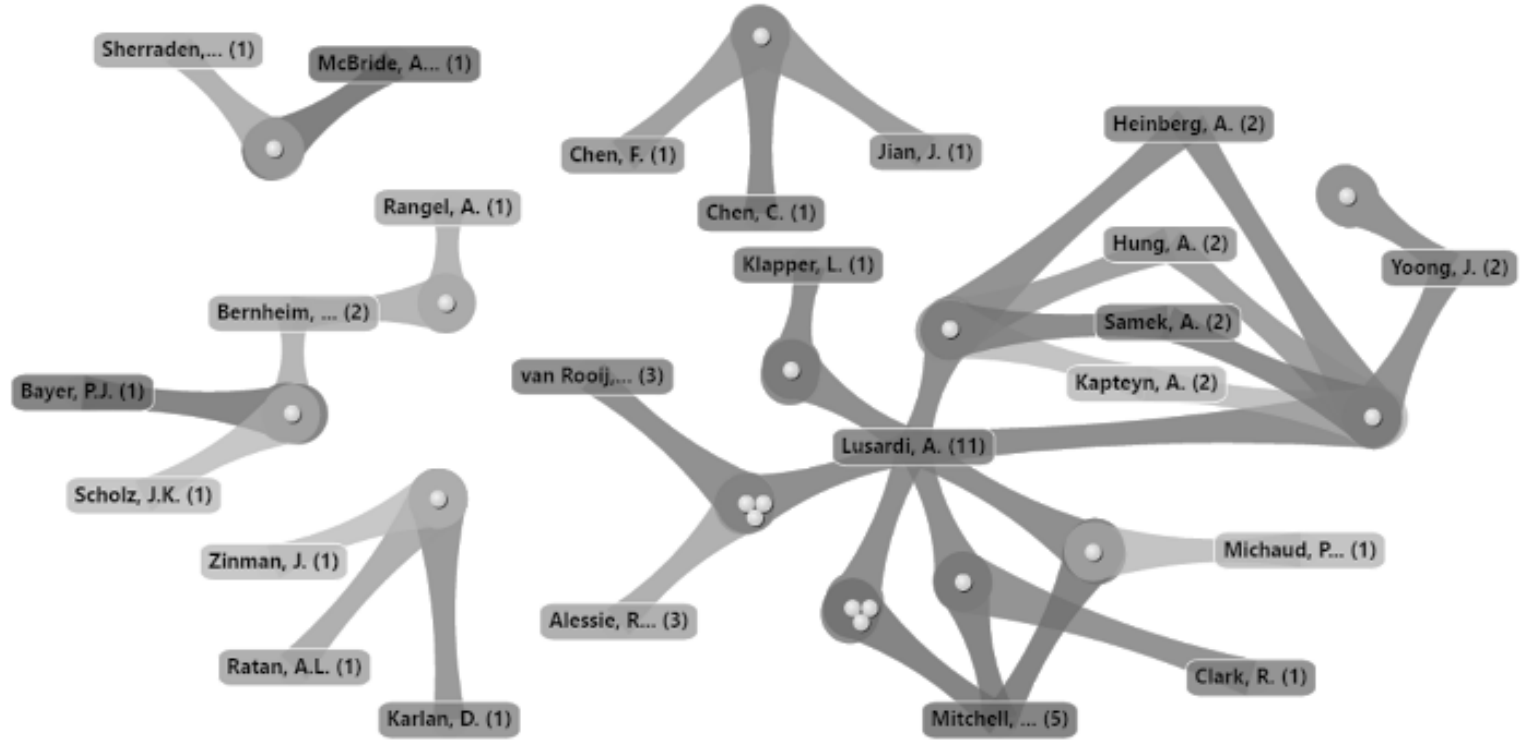

Figura 7. Aduna de colaboración entre autores para producciones científicas. Fuente: Autores utilizando el software Vantage Point.

En tal sentido, destacan las colaboraciones y producciones realizadas por autores como Lusardi y Mitchell, quienes trabajaron juntos en la realización de 5 de los artículos obtenidos en la búsqueda. Estos autores resaltan en el eje central de la principal red de colaboraciones de producciones científicas que se logra identificar, a partir del gráfico de la aduna, lo que confirma su relevancia dentro de la búsqueda.

A continuación se presentan los resultados de la revisión de literatura realizada a partir de los 53 artículos seleccionados mediante lectura crítica que fueron considerados de mayor relevancia y afinidad a la temática de investigación. 


\section{Análisis de la revisión de literatura}

Dadas las políticas y requisitos educativos que continúan desarrollándose para la educación en materia de finanzas personales, diversos autores coinciden en que es indispensable atender a esta necesidad e incorporar al currículo escolar programas que permitan educar económica y financieramente a los estudiantes, con un proceso que inicie en la escuela primaria, se mantenga en la escuela secundaria y continúe en la universidad, con el objetivo de garantizar así un aprendizaje continuo (Amagir et al., 2018; Parrish y Servon, 2006). En tal sentido, algunos autores han decidido evaluar la efectividad de dichos programas y verificar si en efecto la implementación de la educación financiera en el currículo escolar genera algún efecto sobre los conocimientos y comportamientos financieros de las personas.

Hasta ahora, los hallazgos encontrados en estos estudios revelan en su mayoría que efectivamente los comportamientos financieros (es decir, la habilidad, la actitud y el conocimiento) de los estudiantes de secundaria se ven afectados positivamente por la implementación de la educación financiera impartida a través del currículo escolar (Aprea et al., 2016; Cordero y Pedraja, 2019; Lusardi y Mitchell, 2007; Supanantaroek et al., 2017; Tennyson y Nguyen, 2001), si bien su relevancia varía según el enfoque, las características y el tipo de curso impartido (Förster et al., 2019).

\section{Características de los programas de educación financiera}

En primer lugar, según algunos autores, como Parrish y Servon (2006), que se han centrado específicamente en aspectos como la relevancia del tipo de enfoques que se han utilizado en la implementación de estos programas, a nivel de la escuela secundaria la educación financiera podría implementarse utilizando un enfoque independiente, es decir, como una materia que proporcione un tipo específico de educación, ya que de esta forma se obtienen mejores resultados en los comportamientos financieros de los estudiantes. En contraste, otros autores plantean que, a nivel de la escuela secundaria, el uso del enfoque transversal, es decir, como parte del plan de estudios de otras materias, es la verdadera clave que conduce a contribuir de manera significativa en la adquisición de conocimientos financieros (Cordero y Pedraja, 2019). No obstante, y según los hallazgos encontrados por Walstad et al. (2010), los programas para estudiantes de secundaria pueden mejorar significativamente la educación financiera de los jóvenes, independientemente del tipo de enfoque que estén aplicando.

De acuerdo con esto, Tennyson y Nguyen (2001) llegaron a la conclusión de que incorporar la educación económica y financiera en las instituciones educativas puede ser eficaz para aumentar el conocimiento de los estudiantes independientemente del tipo de enfoque, pero solo si el programa incluye una exposición significativa a los conceptos requeridos de finanzas personales, de acuerdo con las necesidades y realidad de la población, y adicionalmente, el tema se enseñe de una manera estructurada y durante el transcurso de varios años escolares (Van Campenhout, 2015).

\section{Comportamientos financieros}

Por otra parte, la literatura reciente ha demostrado que la implementación de estos programas en el currículo escolar genera efectos positivos sobre algunos de los comportamientos financieros de los jóvenes en educación secundaria (Cordero y Pedraja, 2019; Förster et al., 2019; Zhu, 2019).

En tal sentido, la mayor parte de estas investigaciones sugiere que las personas que participan en un plan de estudios financiero en la escuela secundaria mejoran su comportamiento de ahorro y muestran un mayor patrimonio neto cuando son adultos (Barua et al., 2018; Bernheim et al., 2001; Jayaraman et al., 2018; Supanantaroek et al., 2017). Del mismo modo, varios autores brindan evidencia clara de que la educación financiera tiene efectos significativos sobre el comportamiento de ahorro para la jubilación y su planificación (Cordero y Pedraja, 2019; Jayaraman et al., 2018; Lusardi y Mitchell, 2007).

Además, los estudios que evalúan los impactos de la inclusión financiera en el bienestar de los jóvenes evidencian resultados económicos, sociales y de salud positivos, incluidos la titularidad de una cuenta de ahorro y mayores niveles de bienestar económico y financiero (Chowa y Despard, 2014; Chowa y Ansong, 2010), así como una relación positiva entre el ahorro, el rendimiento académico y las expectativas de los estudiantes (Aprea et al., 2016).

Por otra parte, existen investigaciones que demuestran que la adquisición de conocimiento financiero está asociada, aunque en menor medida, con el desarrollo de otros comportamientos financieros, tales como contratar hipotecas, realizar préstamos con tasas de interés más bajas y comisiones (Disney y Gathergood, 2013; Lusardi y Tufano, 2015), participar en el mercado de valores, diversificar la cartera o la tendencia a evitar el sobreendeudamiento (Lusardi y Tufano, 2015; Van Rooij et al., 2011).

\section{Conclusiones}

A partir de los resultados obtenidos de las diferentes investigaciones consultadas, se logra identificar que el 
principal comportamiento que podrían desarrollar o cambiar los jóvenes mediante la implementación de los programas de educación económica y financiera es el relacionado con el ahorro y la planificación para la jubilación. Así mismo, se reconoce que en efecto estos programas pueden mejorar positiva y significativamente la educación financiera de los jóvenes, independientemente del tipo de enfoque que se esté aplicando, pero solo si el programa de educación financiera implementado incluye una exposición significativa de los conceptos requeridos de finanzas personales, y adicionalmente, el tema se enseñe de una manera estructurada y durante el transcurso de varios años escolares.

Por otra parte, a través del análisis bibliométrico, se puede confirmar cómo en el ámbito mundial se refleja una escasez de estudios que abordan esta temática de investigación, resaltando principalmente a Europa, África y América Latina. Se reconoce entonces la falta de educación económica y financiera de la población y la necesidad de encausar esfuerzos desde los gobiernos y las instituciones educativas para promover el diseño e implementación de estrategias enfocadas en mejorar los niveles de alfabetización financiera.

Bajo este escenario, se desarrolla un proyecto que busca aportar al proceso pedagógico de educación económica y financiera de los jóvenes colombianos, incentivando el desarrollo de estilos de vida sostenibles en materia de finanzas personales, mediante el uso de una herramienta gamificada, acorde a la realidad y necesidades de la población. Dicho proyecto se titula "Educación financiera gamificada como estrategia didáctica para el desarrollo de estilos de vida sostenibles".

\section{Agradecimientos}

Agradecemos a la Universidad Industrial de Santander por facilitarnos los escenarios para la realización de esta investigación. De igual forma, a la profesora Martha Liliana Torres Barreto, directora del Grupo de Investigación Finance \& Management y del Laboratorio Galea de esa institución, por su apoyo y comentarios para la construcción de este manuscrito.

\section{Referencias}

Acosta-Medina, J. K., Torres-Barreto, M. L., AlvarezMelgarejo, M. y Paba-Medina, M. C. (2020). Gamificación en el ámbito educativo: Un análisis bibliométrico. I+ D Revista de investigaciones, 15(1), 28-36.

Ahmad, Z., Simun, M. y Masuod, M. S. (2014). Determinants of Financial Behaviours among Malaysians. Indonesian Capital Market Review, 2(2), 121-132. https://doi.org/10.21002/icmr.v2i2.3663
Amagir, A., Groot, W., Maassen van den Brink, H., y Wilschut, A. (2018). A review of financial-literacy education programs for children and adolescents. Citizenship, Social and Economics Education, 17(1), 56-80. https:// doi.org/10.1177/2047173417719555

Aprea, C., Wuttke, E., Breuer, K., Koh, N. K., Davies, P., GreimelFuhrmann, B. y Lopus, J. S. (2016). International Handbook of Financial Literacy. International Handbook of Financial Literacy, 1-713. https://doi. org/10.1007/978-981-10-0360-8

Arango, L. C. (2018). Educación superior: la alfabetización en géneros discursivos. Educação \& Realidade, 43(2), 629-651.

Asobancaria. (2016). Programas de Educación Financiera en Latinoamérica : un énfasis en el caso colombiano. Semana Económica 2016, 1-13.

Atkinson, A. y Messy, F. A. (2012). Measuring Financial Literacy: Results of the OECD / International Network on Financial Education (INFE) Pilot Study. OECD Working Papers on Finance, Insurance and Private Pensions. https://doi.org/10.1111/j.17456606.2010.01170.x

Barua, R., Koh, B. y Mitchell, O. S. (2018). Does Financial Education Enhance Financial Preparedness? Evidence from a Natural Experiment in Singapore. Journal of Pension Economics and Finance, 17(3), 254277. https://doi.org/10.1017/S1474747217000312

Bernheim, B. D., Garrett, D. M. y Maki, D. M. (2001). Education and Saving: The Long-Term Effects of High School Financial Curriculum Mandates. Journal of Public Economics, 80(3), 435-465.

Cameron, M. P., Calderwood, R., Cox, A., Lim, S. y Yamaoka, M. (2014). Factors Associated with Financial Literacy Among High School Students in New Zealand. International Review of Economics Education, 16(PA), 12-21. https://doi.org/10.1016/j.iree.2014.07.006

Chowa, G. A. N. y Despard, M. R. (2014). The Influence of Parental Financial Socialization on Youth's Financial Behavior: Evidence from Ghana. Journal of Family and Economic Issues, 35(3), 376-389. https://doi. org/10.1007/s10834-013-9377-9

Chowa, G. y Ansong, D. (2010). Youth and Savings in AssetsAfrica. Children and Youth Services Review, 32(11), 1591-1596.

Cordero, J. M. y Pedraja, F. (2019). The Effect Of Financial Education Training on the Financial Literacy of Spanish Students in PISA. Applied Economics, 51(16), 1679-1693. https://doi.org/10.1080/00036846.2018. 1528336

Disney, R. y Gathergood, J. (2013). Financial Literacy and Consumer Credit Portfolios. Journal of Banking \& Finance, 37(7), 2246-2254.

Ergün, K. (2018). Financial Literacy Among University Students: A Study in Eight European Countries. International Journal of Consumer Studies, 42(1), 2-15. https://doi.org/10.1111/ijcs.12408

Fabris, N. y Luburic, R. (2016). Financial Education of 
Children and Youth. Journal of Central Banking Theory and Practice, 5(2), 65-79. https://doi.org/10.1515/ jcbtp-2016-0011

Förster, M., Happ, R. y Walstad, W. B. (2019). Relations Between Young Adults' Knowledge and Understanding, Experiences, and Information Behavior in Personal Finance Matters. Empirical Research in Vocational Education and Training, 11(1). https://doi.org/10.1186/s40461-019-0077-z

Jang, K., Hahn, J. y Park, H. J. (2014). Comparison of Financial Literacy Between Korean and U.S. High School Students. International Review of Economics Education, 16(Part A), 22-38. https://doi.org/10.1016/j. iree.2014.07.003

Jayaraman, J. D., Jambunathan, S. y Adesanya, R. (2018). Financial Literacy and Classroom Practices Among Early Childhood and Elementary Teachers in India and the US. Education 3-13, 4279. https://doi.org/10.1 080/03004279.2018.1533030

Liu, C. L., Chang, D. F. y Chuang, C. M. (2019). Investigation of the Financial Literacy Among High School Students. ICIC Express Letters, Part B: Applications, 10(5), 387393. https://doi.org/10.24507/icicelb.10.05.387

Lusardi, A. y Mitchell, O. (2011). Financial Literacy Around the World: An Overview. Journal of Pension Economics \& Finance, 10(4), 497-508.

Lusardi, A. y Mitchell, O. (2014). The Economic Importance of Financial Literacy: Theory and Evidence. Journal of Economic Literature, 52(1), 5-44.

Lusardi, A. y Mitchell, O. S. (2007). Financial Literacy and Retirement Planning: New Evidence from the Rand American Life Panel. In Michigan Retirement Research Center Research Paper (No. 2007/33). https://doi. org/10.1017/CBO9781107415324.004

Lusardi, A. y Tufano, P. (2015). Debt Literacy, Financial Experiences, and Overindebtedness. Journal of Pension Economics \& Finance, 14(4), 332-368.

OCDE/INFE. (2019). Declaración en apoyo a las políticas de educación financiera por los miembros de la OCDE/INFE de America Latina y el Caribe. https://doi. org/10.1017/CBO9781107415324.004

OECD. (2005). Improving Financial Literacy: Analysis of Issues and Policies. https://read.oecd-ilibrary.org/ finance-and-investment/improving-financial-literacy_ fmt-v2005-art11-en\#page5

OECD. (2014). PISA 2012 Results: Students and Money: Financial Literacy Skills for the 21st Century (Volume VI). In Pisa, OECD Publishing. https://doi. org/10.1787/9789264208094-en

Parrish, L. y Servon, L. (2006). Equipping Families for their Financial Futures: Policy Recommendations to Improve Financial Literacy. New America Foundation.

Supanantaroek, S., Lensink, R. y Hansen, N. (2017). The Impact of Social and Financial Education on Savings Attitudes and Behavior Among Primary School Children in Uganda. Evaluation Review, 41(6), 511541. https://doi.org/10.1177/0193841X16665719
Tennyson, S. y Nguyen, C. (2001). State Curriculum Mandates and Student Knowledge of Personal Finance. Journal of Consumer Affairs, 35(2), 241-262.

Van Campenhout, G. (2015). Revaluing the Role of Parents as Financial Socialization Agents in Youth Financial Literacy Programs. Journal of Consumer Affairs, 49(1), 186-222.

Van Rooij, M. C., Lusardi, A. y Alessie, R. J. (2011). Financial Literacy and Retirement Planning in the Netherlands. Journal of Economic Psychology, 32(4), 593-608.

Walstad, W. B., Rebeck, K. y MacDonald, R. A. (2010). The Effects of Financial Education on the Financial Knowledge of High School Students. Journal of Consumer Affairs, 44(2), 336-357.

Zhu, A. Y. F. (2019). Impact of Financial Education on Adolescent Financial Capability: Evidence from a Pilot Randomized Experiment. Child Indicators Research. https://doi.org/10.1007/s12187-019-09704-9 